\title{
Concordant sequences and integral-valued entire functions
}

\author{
by \\ Jonathan Pila (Melbourne) and \\ Fernando Rodriguez Villegas (Austin, Tex.)
}

A classic theorem of Pólya shows that the function $2^{z}$ is the "smallest" integral-valued entire transcendental function. A variant due to Gel'fond applies to entire functions taking integral values on a geometric progression of integers, and Bézivin has given a generalization of both results. We give a sharp formulation of Bézivin's result together with a further generalization.

1. Introduction. In a classic paper [15], Pólya showed that the growth of an entire transcendental function places restrictions on its integral values.

Let $f$ be an entire function and denote by $M(f, r)$ the maximum of $|f(z)|$ for $|z| \leq r$. Suppose that $f(n)$ is an integer for each non-negative integer $n$. Pólya showed in [15] that if

$$
\lim _{r \rightarrow \infty} \frac{M(f, r) \sqrt{r}}{2^{r}}=0
$$

then $f$ is a polynomial.

The obtrusive factor $\sqrt{r}$ was removed by Hardy [11]. The result was then further sharpened by Pólya [16] to the following. Let $f$ be an entire function such that $f(n)$ is an integer for each non-negative integer $n$. Suppose that

$$
\limsup _{r \rightarrow \infty} \frac{M(f, r)}{2^{r}}<1 .
$$

Then $f$ is a polynomial.

Thus the function $2^{z}$ is the "smallest" transcendental entire function taking integral values on the set $\mathbb{N}=\{0,1, \ldots\}$.

The commentary [3] by Boas in Pólya's collected works indicates some of the many lines of research stimulated by Pólya's result.

A variation on Pólya's result for geometric progressions was obtained by Gel'fond [8] (see also [9, §2.3.4]). The result, slightly reformulated (Gel'fond

1991 Mathematics Subject Classification: Primary 11J99 
considered geometric progressions $a, a^{2}, a^{3}, \ldots$ but it is more convenient for us to begin the sequence with $a^{0}$ ), is as follows. Let $a \in \mathbb{Z},|a| \geq 2$. Let $f$ be an entire function taking integral values on the set $X_{a}=\left\{1, a, a^{2}, \ldots\right\}$ and suppose that

Then $f$ is a polynomial.

$$
\lim _{r \rightarrow \infty} \frac{M(f, r)}{\exp \left((\log r)^{2} /(4 \log a)\right)}=0 .
$$

Gel'fond exhibited a function $T_{a}$ that plays the role of the "smallest" $X_{a}$-integral transcendental entire function and showed that

$$
M\left(T_{a}, r\right)=O\left(\exp \left(\frac{(\log r)^{2}}{4 \log a}\right)\right) .
$$

Thus Gel'fond's result is not quite as sharp at the boundary as that of Pólya-Hardy above. We describe the construction of $T_{a}$ below.

A result of Bézivin [1] generalizes both of these theorems. It is convenient to deal with sequences rather than sets, and indeed with sequences $X=$ $\left\{x_{0}, x_{1}, \ldots\right\}$ that do not contain any repetitions: $x_{i}=x_{j}$ if and only if $i=j$. We will call such a sequence (finite or infinite) proper. Bézivin considers sequences $X=\left\{x_{0}, x_{1}, \ldots\right\} \subset \mathbb{Z}$ that are the (infinite) orbits obtained by the iteration of a univariate polynomial $P$ with integral coefficients:

$$
X_{P, x_{0}}=\left\{x_{0}, x_{1}, \ldots \mid x_{0} \in \mathbb{Z}, x_{i+1}=P\left(x_{i}\right), i=0,1, \ldots\right\} .
$$

The case considered by Pólya arises from the choice $P(x)=x+1, x_{0}=0$, and the case considered by Gel'fond from $P(x)=a x,|a| \geq 2, x_{0}=1$. As a further instance, the choice $P(x)=x^{b}, b \geq 2, x_{0}=a \geq 2$ yields a result of Pólya-Gel'fond type for the set

$$
X_{a, b}=\left\{a^{b^{n}} \mid n=0,1, \ldots\right\} .
$$

Let $P$ be of degree $d \geq 2$ and $x_{0} \in \mathbb{Z}$ with $X=X_{P, x_{0}}$ proper. Bézivin exhibits a constant $\lambda_{0}=\lambda_{0}\left(P, x_{0}\right)$ and proves the following result. Let $f(z)$ be an entire function that is integral-valued on $X$. Suppose that $\lambda>\lambda_{0}$ and that, for all sufficiently large $r$,

$$
M(f, r) \leq \exp \left(\frac{\log r \log \log r-\log r \log \log \log r}{\log d}-\lambda \log r\right) .
$$

Then $f$ is a polynomial.

Bézivin exhibits a transcendental entire function $T_{X}$ that is integralvalued on $X$ and satisfies

$$
M\left(T_{X}, r\right) \leq \exp \left(\frac{\log r \log \log r-\log r \log \log \log r}{\log d}+O(\log r)\right)
$$

for all sufficiently large $r$. Thus the result is best possible in respect of the main terms of the order of growth. The construction of $T_{X}$ is described below. 
We prove a sharp version of Bézivin's result in which the growth of the $X$-integral function $f$ is compared directly with the growth of $T_{X}$. Our result shows that $T_{X}$ is the "smallest" transcendental entire function that is integral-valued on $X$ in the sense of the above mentioned result of Pólya [16]. Our result also applies to a somewhat more general class of sequences $X$; namely, to sequences that enjoy two properties that we now proceed to describe.

The first property is of an arithmetic nature. A sequence $X=\left\{x_{0}, x_{1}, \ldots\right\}$ of integers will be called concordant if, for all non-negative integers $i, j, d$, we have

$$
x_{i} \equiv x_{j} \bmod d \Rightarrow x_{i+1} \equiv x_{j+1} \bmod d .
$$

Sequences of the form $X_{P, x_{0}}$ are evidently concordant. More generally, a concordant sequence results from the iteration (where possible) of any pseudopolynomial as defined by Hall [10]; that is, a function $T: \mathbb{N} \rightarrow \mathbb{Z}$ satisfying $T(n+k) \equiv T(n) \bmod k$ for all non-negative integers $n, k$. (To enable iteration one would want $T: \mathbb{N} \rightarrow \mathbb{N}$ or $T$ defined on all $\mathbb{Z}$.) There exist pseudopolynomials that are not polynomials; there also exist pseudopolynomials that are polynomials but that do not have integral coefficients. For further information on pseudopolynomials see [10], [13].

Concordant sequences may be composed preserving the concordant property. If $X=\left\{x_{0}, x_{1}, \ldots\right\}$ and $Y=\left\{y_{0}, y_{1}, \ldots\right\}$ are sequences with $y_{n} \geq 0$ for all $n$ we define

$$
X_{Y}=Z=\left\{z_{0}, z_{1}, \ldots\right\}, \quad z_{n}=x_{y_{n}}, \quad n=0,1, \ldots
$$

The following proposition is proved in Section 5 .

Proposition 1.1. Let $X=\left\{x_{0}, x_{1}, \ldots\right\}$ and $Y=\left\{y_{0}, y_{1}, \ldots\right\}$ be concordant sequences of integers and suppose that $0 \leq y_{0}<y_{1}<\ldots$ Then $X_{Y}$ is concordant.

If $X, Y$ are of the form $X_{P, x_{0}}$ with $P$ a polynomial then $X_{Y}$ will not in general be of this form; for example, if $X=X_{a}$ and $Y=X_{b, c}$ then $X_{Y}$ consists of the points

$$
a^{b^{c^{n}}}, \quad n=0,1,2, \ldots
$$

The second property that we require is of an analytic nature and entails that the sequence be very sparse. A sequence $X=\left\{x_{0}, x_{1}, \ldots\right\}$ of complex numbers will be called diffuse if

$$
\lim _{n \rightarrow \infty}\left|x_{n}\right|=\infty, \quad \lim _{n \rightarrow \infty} \frac{\left|x_{n+1}\right|^{n+1}}{\left|x_{n}\right|^{2 n}}=\infty .
$$

We prove in Section 3 that, if $P$ has degree 2 or greater, then the sequence $X_{P, x_{0}}$, if proper, is diffuse. In Section 5 we prove that any subsequence of a diffuse sequence is diffuse. 
Given a diffuse concordant sequence $X$ of integers we define below an entire function $T_{X}$, following the constructions of Gel'fond and Bézivin, that plays the role of the "smallest" transcendental entire function that is integral-valued on $X$. The following theorem is proved in Section 4 .

TheOREM 1.2. Let $X$ be a diffuse concordant sequence of integers. Let $f$ be an entire function that takes integral values on $X$ and satisfies

$$
\limsup _{r \rightarrow \infty} \frac{M(f, r)}{M\left(T_{X}, r\right)}<1 .
$$

Then $f$ is a polynomial.

The proof of Theorem 1.2 follows the same line of proof as Pólya [15], Gel'fond [8] and Bézivin [1]. A certain sequence of polynomials associated with $X$ plays a fundamental role. For Pólya, these are the polynomials

$$
\phi_{0}(x)=1, \quad \phi_{j}(x)=\frac{x(x-1) \ldots(x-j+1)}{j !}, \quad j=1,2, \ldots
$$

These polynomials have the following properties: $\phi_{n}$ has degree $n$, vanishes at the points $0,1, \ldots, n-1$ and takes the value 1 at $n$. They have the additional property of taking integral values on $\mathbb{N}$ (indeed on $\mathbb{Z}$ ). An analogous sequence $\phi_{X, n}$ of polynomials may be associated with any proper (infinite) sequence $X=\left\{x_{0}, x_{1}, \ldots\right\}$ of complex numbers. They are uniquely determined by the properties that $\phi_{X, n}$ has degree $n$, vanishes at $x_{0}, x_{1}, \ldots, x_{n-1}$ and satisfies $\phi_{X, n}\left(x_{n}\right)=1$.

A sequence $X$ of integers will be called a parade if each of the polynomials $\phi_{X, n}$ has the additional property of being integral-valued on $X$. We will refer to the polynomials $\phi_{X, n}$ as the attendant polynomials of the parade $X$. Bézivin [1] proves that the sequences $X_{P, x_{0}}$ are parades. We prove the following generalization in Section 2.

Proposition 1.3. Let $X$ be a proper concordant sequence. Then $X$ is a parade.

Let us mention also that while concordant sequences provide many examples of parades, the sequence $x_{n}=n^{2}, n=0,1,2, \ldots$, is a parade but is not concordant. This sequence is also not diffuse.

For a parade $X$ that is sufficiently sparse the "smallest" $X$-integral transcendental entire function is constructed by simply adding up the sequence of attendant polynomials. One easily proves (see Section 3) the following.

Proposition 1.4. Let $X$ be a diffuse sequence of complex numbers. Then the series

$$
T_{X}(z)=\sum_{n=0}^{\infty} \phi_{X, n}(z)
$$


converges absolutely for all complex $z$ and determines an entire function $T_{X}$.

We will call $T_{X}$ the envelope function of $X$. When $X=\mathbb{N}$, i.e. Pólya's situation, the series converges to $2^{z}$ only at non-negative integers. So we will define the envelope function of $\mathbb{N}$ to be the function $2^{z}$. For $P=x+k, X=$ $X_{P, x_{0}}$ we can similarly define $T_{X}(z)=2^{\left(z-x_{0}\right) / k}$. Since a very sharp result nevertheless obtains in the case $X=\mathbb{N}$, it might be hoped that sparsity hypotheses such as our notion of diffusity could be dispensed with in the formulation of results of the type of Theorem 1.2. However if the sum of attendant polynomials does not converge to an entire function it is not clear in general how to interpolate the values on $X$ by an entire function in an appropriate way to define $T_{X}$.

Various authors have investigated the structure of entire functions that are integral-valued but grow faster than $2^{z}$. A result of Pólya [16] in this direction is as follows. Let $k$ be a positive number. Suppose that $f$ is an integral-valued entire function and that $M(f, r) r^{-k} / 2^{r}$ is bounded as $r \rightarrow$ $\infty$. Then there are polynomials $P, Q$ such that $f(z)=P(z) 2^{z}+Q(z)$. Selberg [18] obtains the same conclusion under the weaker assumption

$$
\limsup _{r \rightarrow \infty} \frac{\log M(f, r)}{r} \leq \log 2+\frac{1}{1500} .
$$

Pisot [14] shows that if

$$
\limsup _{r \rightarrow \infty} \frac{\log M(f, r)}{r}<0.843 \ldots
$$

then $f$ is of the form

$$
\alpha_{1}^{z} P_{1}(z)+\alpha_{2}^{z} P_{2}(z)+\ldots+\alpha_{h}^{z} P_{h}(z)
$$

where $\alpha_{1}, \ldots, \alpha_{h}$ are algebraic integers. See also related results of Buck [5] and Robinson [17].

As remarked by Buck [5], any structure results of these kinds must be limited to functions growing slower than the function $\sin (\pi z)$ : if $g$ is any entire function then $g(z) \sin (\pi z)$ is integral-valued.

It would be interesting to pursue analogous results for other parades. As far as we are aware, none are known even for the case of geometric progressions.

For a diffuse parade $X$ the canonical product

$$
H_{X}(z)=\prod_{n=0}^{\infty}\left(1-\frac{z}{x_{n}}\right)
$$

is entire, and any investigation of the structure of $X$-integral entire functions would be confined to functions whose growth rate is between those of $T_{X}$ and $H_{X}$. 
Bézivin [2] considers integral-valued entire functions $f$ on very general subsequences $X$ of geometric progressions $X_{a}$. The sequences considered are not in general parades. He proves under quite general hypotheses that if $f$ is $X$-integral and

$$
\limsup _{r \rightarrow \infty} \frac{\log M(f, r)}{\log M\left(H_{X}, r\right)}<1
$$

then $f$ is a polynomial.

Thus any interesting integral-valued entire functions and structure of such functions along the lines of the above mentioned results for such sequences $X$ would need to occur among functions $f$ that do not satisfy the above condition relative to $H_{X}$ but that nevertheless grow slower than $H_{X}$.

In view of Proposition 1.1 and the properties of diffuse sequences described above, Theorem 1.2 applies to any concordant subsequence of proper sequences of the form $X_{P, x_{0}}$ where the degree of $P$ is at least 2. Hence in particular it applies to the sequences

$X_{a, b, c}=\left\{a^{b^{c^{n}}} \mid n=0,1,2, \ldots\right\}, \quad X_{a, b, c, d}=\left\{a^{b^{c^{d^{n}}}} \mid n=0,1,2, \ldots\right\}, \quad$ etc.

The results of Bézivin [2] also apply to these sequences. Thus in these cases one has at least the function $T_{X}$ in the growth-rate range of interest.

The remainder of the paper, Section 6 , is devoted to establishing some properties of envelope functions. For the functions $T_{a}$ of Gel'fond we get a combinatorial expression for the Taylor coefficients by establishing a recurrence relation satisfied by $T_{a}$. We also show that the envelope function of any diffuse increasing parade shares with the function $2^{z}$ the property that all but finitely many Taylor coefficients are positive.

Acknowledgements. We are grateful to J. FitzGerald, K. Ford, B. Poonen, I. Rivin and J. Tate for discussions and assistance at various junctures. The first author thanks P. Sarnak and the Mathematics Department of Princeton University for their hospitality during the visit that occasioned the present collaboration. We are also most grateful to the referee for suggesting several substantial improvements to this paper.

2. Concordant sequences and parades. In this section we prove Proposition 1.3 in a general setting.

Let $R$ be a commutative ring with 1 . A sequence $X=\left\{x_{n}\right\} \subset R, n \in$ $\mathbb{Z}_{\geq 0}$, will be called proper if the $x_{n}$ 's are all distinct, and concordant if it is proper and for every $i, j \in \mathbb{Z}_{\geq 0}$ and every ideal $I \subset R$ we have

$$
x_{i} \equiv x_{j} \bmod I \Rightarrow x_{i+1} \equiv x_{j+1} \bmod I \text {. }
$$

The sequences $X_{P, x_{0}}$ are easily seen to be concordant sequences in $\mathbb{Z}$ : if $x_{i} \equiv x_{j} \bmod d$ then $P\left(x_{i}\right) \equiv P\left(x_{j}\right) \bmod d$. In Section 5 we construct some further natural examples of concordant sequences in $\mathbb{Z}$. 
Given a concordant sequence $X \subset R$ and an ideal $I \subset R$ consider the sequence $\bar{x}_{n}=x_{n} \bmod I$. Let $i \in \mathbb{Z}_{\geq 0}$ be the smallest index (if any such indices exist) so that there exists a $j^{\prime} \in \mathbb{Z}_{\geq 0}$ such that $i<j^{\prime}$ and $\bar{x}_{j^{\prime}}=\bar{x}_{i}$. Let $j$ be the smallest such $j^{\prime}$ and set $\delta=j-i \in \mathbb{N}$. It is not hard to see that $\bar{x}_{n}$ is then of the form

$$
\bar{x}_{0}, \bar{x}_{1}, \ldots, \bar{x}_{i-1}, \quad \bar{x}_{i}, \ldots, \bar{x}_{i+\delta-1}, \quad \bar{x}_{i}, \ldots, \bar{x}_{i+\delta-1}, \quad \ldots
$$

with no repetitions other than those explicitly indicated. More precisely,

$$
\bar{x}_{j}=\bar{x}_{j^{\prime}} \quad \text { if and only if } i \leq j, j^{\prime} \text { and } j \equiv j^{\prime} \bmod \delta .
$$

From now on we assume that $R$ is a domain and let $K$ be its field of fractions. Given a proper sequence $X$ in $R$ and two positive integers $m, n \in \mathbb{N}$ with $m>n$ we define

$$
\left(\begin{array}{c}
m \\
n
\end{array}\right)_{X}=\frac{\left(x_{m}-x_{0}\right)\left(x_{m}-x_{1}\right) \ldots\left(x_{m}-x_{n-1}\right)}{\left(x_{n}-x_{0}\right)\left(x_{n}-x_{1}\right) \ldots\left(x_{n}-x_{n-1}\right)} \in K .
$$

If $R=\mathbb{Z}$ and $x_{n}=n$ for all $n \in \mathbb{Z}_{\geq 0}$ then, of course, this is just the usual binomial coefficient $\left(\begin{array}{l}m \\ n\end{array}\right)$, which explains our choice of notation. Our goal is to prove the following.

Proposition 2.1. Let $R$ be an integrally closed domain and let $X$ be a proper concordant sequence in $R$. Suppose that $m, n \in \mathbb{Z}$ with $m>n$. Then

$$
\left(\begin{array}{c}
m \\
n
\end{array}\right)_{X} \in R
$$

Proof. Let $A$ be a valuation ring in $K$ containing $R$. That is, $A$ is a subring of $K$ containing $R$ and equipped with a valuation $v: A \backslash\{0\} \rightarrow V$, where $V$ is a totally ordered group. Fix $m, n \in \mathbb{N}$ with $m>n$. For any $k \in V$ we define

$$
\begin{gathered}
N(k)=\#\left\{j \mid 0 \leq j<n, v\left(x_{m}-x_{j}\right) \geq k\right\}, \\
D(k)=\#\left\{j \mid 0 \leq j<n, v\left(x_{n}-x_{j}\right) \geq k\right\} .
\end{gathered}
$$

It is clear that $N, D: V \rightarrow \mathbb{N}$ are decreasing step functions. More precisely, let $k_{1}<\ldots<k_{s} \in V$ be an ordered enumeration of the set $\left\{v\left(x_{m}-x_{i}\right)\right\} \cup$ $\left\{v\left(x_{n}-x_{i}\right)\right\}$, where $i=0, \ldots, n-1$. Then $N$ and $D$ are constant on each of the sets $\left\{k \in V \mid k \leq k_{1}\right\},\left\{k \in V \mid k_{j-1}<k \leq k_{j}\right\}$ for $j=2, \ldots, s$, and $\left\{k \in V \mid k_{s}<k\right\}$. To simplify the notation we will let $N_{j}=N\left(k_{j}\right)$ and $D_{j}=D\left(k_{j}\right)$ for $j=1, \ldots, s-1$. We have

$$
\mathrm{N}=v\left(\left(x_{m}-x_{0}\right)\left(x_{m}-x_{1}\right) \ldots\left(x_{m}-x_{n-1}\right)\right)=\sum_{j=1}^{s-1} k_{j}\left(N_{j}-N_{j+1}\right)+k_{s} N_{s}
$$

and

$$
\mathrm{D}=v\left(\left(x_{n}-x_{0}\right)\left(x_{n}-x_{1}\right) \ldots\left(x_{n}-x_{n-1}\right)\right)=\sum_{j=1}^{s-1} k_{j}\left(D_{j}-D_{j+1}\right)+k_{s} D_{s} .
$$


By summation by parts,

$$
\mathrm{N}=k_{1} N_{1}+\sum_{j=2}^{s}\left(k_{j}-k_{j-1}\right) N_{j}, \quad \mathrm{D}=k_{1} N_{1}+\sum_{j=2}^{s}\left(k_{j}-k_{j-1}\right) D_{j} .
$$

Fix $k \in V$ and let $I \subset A$ be the ideal $\{a \in A \mid v(a) \geq k\}$. Also let, as before, $\bar{x}_{n}=x_{n} \bmod I$. Since $x_{n}$ is concordant our above description of $\bar{x}_{n}$ guarantees the following. Assume first that $i \leq n$ and let $j, j^{\prime}$ be the unique indices $i \leq j, j^{\prime} \leq i+\delta-1$ such that $\bar{x}_{n}=\bar{x}_{j}$ and $\bar{x}_{m}=\bar{x}_{j^{\prime}}$. Then

$$
N(k)= \begin{cases}D(k) & \text { if } j \leq j^{\prime} \\ D(k)+1 & \text { otherwise. }\end{cases}
$$

If $i>n$ then $N(k)=D(k)=0$.

Therefore

$$
v\left(\left(\begin{array}{c}
m \\
n
\end{array}\right)_{X}\right)=\mathrm{N}-\mathrm{D}=k_{1}\left(N_{1}-D_{1}\right)+\sum_{j=2}^{s}\left(k_{j}-k_{j-1}\right)\left(N_{j}-D_{j}\right)
$$

and

$$
0 \leq v\left(\left(\begin{array}{c}
m \\
n
\end{array}\right)_{X}\right) \leq k_{s}
$$

We have proved that $\left(\begin{array}{c}m \\ n\end{array}\right)_{X}$ is in $A$. Since the valuation ring $A$ was arbitrary, $\left(\begin{array}{c}m \\ n\end{array}\right)_{X}$ is also in the intersection of all such $A$ 's. Since $R$ is an integrally closed domain, the intersection of all valuation rings containing $R$ is $R$ [4, VI, §3, Theorem 3]. This completes the proof.

Proof of Proposition 1.3. The conclusion follows from Proposition 2.1 since

$$
\phi_{X, n}(m)=\left(\begin{array}{c}
m \\
n
\end{array}\right)_{X}
$$

Remark 2.2. Bézivin [1] proves a version of Proposition 2.1 for sequences formed by iteration of a univariate polynomial. One may extract somewhat more information in that case. Let $d$ be a positive integer. Let $a_{0}, a_{1}, \ldots, a_{d}$ be independent indeterminates and set $R=\mathbb{Z}\left[a_{0}, a_{1}, \ldots, a_{d}\right]$. Let $K$ be the quotient field of $R$. For a univariate polynomial $P$ and a non-negative integer $k$ we will denote by $P^{[k]}$ the $k$ th iteration of $P$, with the convention that $P^{[0]}$ is the identity polynomial $P^{[0]}(x)=x$. We now let $P \in R[x]$ be the polynomial

$$
P(x)=\sum_{j=0}^{d} a_{j} x^{j}
$$

The sequence $\left\{P^{[0]}, P^{[1]}, P^{[2]}, \ldots\right\}$ in $R[x]$ is concordant. Proposition 2.1 
implies that, for non-negative integers $n, m$ with $m \geq n$, the quotient

$$
Q_{n, m}=\prod_{k=0}^{n-1} \frac{\left(P^{[m]}-P^{[k]}\right)}{\left(P^{[n]}-P^{[k]}\right)} \in K(x)
$$

is in fact in $R[x]$. Thus for $X=X_{P, x_{0}}$ we have $\phi_{X, n}(m)=Q_{m, n}\left(x_{0}\right)$.

3. Diffuse sequences and granularity. We will call a sequence $X$ of complex numbers semidiffuse if

$$
\lim _{n \rightarrow \infty}\left|x_{n}\right|=\infty, \quad \liminf _{n \rightarrow \infty} \frac{\left|x_{n+1}\right|}{\left|x_{n}\right|}>1, \quad \liminf _{n \rightarrow \infty} \frac{\left|x_{n+1}\right|^{n+1}\left|x_{n-1}\right|^{n-1}}{\left|x_{n}\right|^{2 n}}>1 .
$$

Suppose that $U: \mathbb{Z} \rightarrow \mathbb{Z}$. If $x_{0} \in \mathbb{Z}$ then we can form the sequence of iterates of $x_{0}$ under $U$ :

$$
X_{U, x_{0}}=\left\{x_{0}, x_{1}, \ldots \mid x_{0} \in \mathbb{Z}, x_{i+1}=U\left(x_{i}\right), i=0,1, \ldots\right\} .
$$

Proposition 3.1. Suppose that $U: \mathbb{Z} \rightarrow \mathbb{Z}$ satisfies

$$
\liminf _{|n| \rightarrow \infty} \frac{|U(n)|}{n^{2}}>0
$$

Suppose that $X_{U, x_{0}}$ is proper. Then $X_{U, x_{0}}$ is diffuse.

Proof. Write $X=X_{U, x_{0}}=\left\{x_{0}, x_{1}, \ldots\right\}$. Under our hypotheses there exist positive constants $C, c$ such that

$$
|U(n)| /|n| \geq c|n|
$$

for all $|n|>C$. We may assume $c<1$. Now $\left|x_{n}\right| \rightarrow \infty$ as $n \rightarrow \infty$ because $X$ is proper, hence

$$
\left|x_{n+1}\right| /\left|x_{n}\right|=\left|U\left(x_{n}\right)\right| /\left|x_{n}\right| \geq c\left|x_{n}\right| \geq 3 / c
$$

for all sufficiently large $n$, so that $\left|x_{n}\right| \geq(2 / c)^{n}$ for all sufficiently large $n$. Therefore, for sufficiently large $n$,

$$
\frac{\left|x_{n+1}\right|^{n+1}}{\left|x_{n}\right|^{2 n}}=\frac{\left|U\left(x_{n}\right)\right|^{n+1}}{\left|x_{n}\right|^{2 n}} \geq c^{n+1}\left|x_{n}\right|^{2} \geq 2^{2 n} \rightarrow \infty
$$

as $n \rightarrow \infty$. This completes the proof.

Corollary 3.2. Let $P$ be a polynomial of degree 2 or greater and $x_{0} \in \mathbb{Z}$. Suppose that $X_{P, x_{0}}$ is proper. Then $X_{P, x_{0}}$ is diffuse.

Our interest in the application of Proposition 3.1 is confined to the case where $U$ is a pseudopolynomial. Hall [10] gives a description of the ring of pseudopolynomials and proves that any pseudopolynomial $U$ satisfying $U(n)=O\left(\theta^{n}\right)$ for some $\theta<e-1$ is a polynomial. This result, also found by Ruzsa and Perelli-Zannier, has been variously improved (see [13]). This suggests the following question. Suppose $U$ is a pseudopolynomial that is not a polynomial, and that $X_{U, x_{0}}$ is proper. Is $X_{U, x_{0}}$ diffuse? 
Ford [7] has given a neat argument to show that if $X$ is a proper concordant sequence that is not an arithmetic progression then $\lim _{n \rightarrow \infty}\left|x_{n+1}\right| /\left|x_{n}\right|$ is an integer $\geq 2$, or $\infty$. This raises the question of whether such a sequence is always semidiffuse.

For sequences generated by linear polynomials with non-unit slope we get the following.

Proposition 3.3. Let $P \in \mathbb{Z}[x]$ be of the form $P(x)=a x+h$ with $|a| \geq 2$ and $x_{0} \in \mathbb{Z}$. Suppose that $X_{P, x_{0}}$ is proper. Then $X_{P, x_{0}}$ is semidiffuse.

Proof. Write $X=X_{P, x_{0}}=\left\{x_{0}, x_{1}, \ldots\right\}$. For $a \neq 1$ one has

$$
x_{n}=\frac{h}{1-a}+\left(x_{0}-\frac{h}{1-a}\right) a^{n} .
$$

Thus, with $|a| \geq 2$, we see that $X$ is proper if and only if $\left(x_{0}-\frac{h}{1-a}\right) \neq 0$. The semidiffusity follows from

$$
\lim _{n \rightarrow \infty} \frac{\left|x_{n+1}\right|^{n+1}\left|x_{n-1}\right|^{n-1}}{\left|x_{n}\right|^{2 n}}=\lim _{n \rightarrow \infty} \frac{\left|a^{n+1}\right| n+1\left|a^{n-1}\right|^{n-1}}{\left|a^{n}\right|^{2 n}}=a^{2}>1 .
$$

Lemma 3.4. Let $X$ be a diffuse sequence of complex numbers. Then

$$
\lim _{n \rightarrow \infty} \prod_{k=0}^{n-1}\left(1+\frac{\left|x_{k}\right|}{\left|x_{n}\right|}\right)=1, \quad \lim _{n \rightarrow \infty} \prod_{k=0}^{n-1}\left(1-\frac{\left|x_{k}\right|}{\left|x_{n}\right|}\right)=1 .
$$

Proof. Let $\varepsilon>0$. We will show that there is a non-negative integer $N=N_{\varepsilon}$ such that

$$
\prod_{k=0}^{n-1}\left(1+\frac{\left|x_{k}\right|}{\left|x_{n}\right|}\right) \leq 1+\varepsilon \quad \text { for all } n \geq N .
$$

Choose $Q>1$ such that

$$
\exp \left(\frac{1}{Q-1}\right) \leq \sqrt{1+\varepsilon}
$$

Since $X$ is diffuse we have $\lim _{n \rightarrow \infty}\left|x_{n+1}\right| /\left|x_{n}\right|=\infty$ and we may choose a non-negative integer $M$ such that $\left|x_{n+1}\right| \geq Q\left|x_{n}\right|$ for $n \geq M$. Next choose a positive integer $N$ such that, for all $n \geq N$, we have

$$
\prod_{k=0}^{M-1}\left(1+\frac{\left|x_{k}\right|}{\left|x_{n}\right|}\right) \leq \sqrt{1+\varepsilon}
$$

Let now $n \geq N$. For positive $x$ we have $\log (1+x)<x$. Hence

$$
\prod_{k=0}^{n-1}\left(1+\frac{\left|x_{k}\right|}{\left|x_{n}\right|}\right) \leq\left(\prod_{k=0}^{M-1}\left(1+\frac{\left|x_{k}\right|}{\left|x_{n}\right|}\right)\right) \exp \left(\sum_{k=M}^{n-1} \frac{\left|x_{k}\right|}{\left|x_{n}\right|}\right) \text {. }
$$


The first factor on the right is less than $\sqrt{1+\varepsilon}$ since $n \geq N$. For the second factor we get

$$
\exp \left(\sum_{k=M}^{n-1} \frac{\left|x_{k}\right|}{\left|x_{n}\right|}\right) \leq \exp \left(\sum_{j=1}^{\infty} \frac{1}{Q^{j}}\right)=\exp \left(\frac{1}{Q-1}\right) \leq \sqrt{1+\varepsilon}
$$

giving the required estimate.

A similar computation establishes that

$$
\prod_{k=0}^{n-1}\left(1-\frac{\left|x_{k}\right|}{\left|x_{n}\right|}\right) \geq 1-\varepsilon
$$

for all sufficiently large $n$ since, for sufficiently small positive $x$, we have $\log (1-x) \geq-2 x$.

Lemma 3.5. Let $X=\left\{x_{0}, x_{1}, \ldots\right\}$ be a semidiffuse sequence of complex numbers. Then

$$
\limsup _{n \rightarrow \infty} \prod_{k=0}^{n-1}\left(1+\frac{\left|x_{k}\right|}{\left|x_{n}\right|}\right)<\infty, \quad \liminf _{n \rightarrow \infty} \prod_{k=0}^{n-1}\left(1-\frac{\left|x_{k}\right|}{\left|x_{n}\right|}\right)>0 .
$$

Proof. Since $X$ is semidiffuse we may choose an integer $M$ and a constant $\lambda>1$ such that $\left|x_{n+1}\right| /\left|x_{n}\right| \geq \lambda$ for all $n \geq M$. Choose $N$ such that

$$
\prod_{k=0}^{M}\left(1+\frac{\left|x_{k}\right|}{\left|x_{n}\right|}\right) \leq 2
$$

for all $n \geq N$. For such $n$ we have

$$
\begin{aligned}
\prod_{k=0}^{n-1}\left(1+\frac{\left|x_{k}\right|}{\left|x_{n}\right|}\right) & \leq \prod_{k=0}^{M}\left(1+\frac{\left|x_{k}\right|}{\left|x_{n}\right|}\right) \prod_{k=M}^{n-1}\left(1+\frac{\left|x_{k}\right|}{\left|x_{n}\right|}\right) \\
& \leq 2 \exp \left(\sum_{j=1}^{\infty} \lambda^{-j}\right) \leq 2 \exp \left(\frac{1}{\lambda-1}\right) .
\end{aligned}
$$

A similar computation establishes the non-zero lower bound for the lim inf and completes the proof.

Let $X$ be a sequence of complex numbers. For each non-negative integer $n$ we set

$$
r_{n}=r_{n}(X)=\max \left\{\left|x_{0}\right|,\left|x_{1}\right|, \ldots,\left|x_{n}\right|\right\} .
$$

If $X$ is semidiffuse then, for all sufficiently large $n$, we have $r_{n}=\left|x_{n}\right|$ and $\left|x_{n}\right|<\left|x_{n+1}\right|$.

Proof of Proposition 1.4. We prove that $T_{X}$ is entire under the weaker assumption that $X$ is semidiffuse. Let $U$ be a positive number. Suppose 
$z \in \mathbb{C}$ with $|z| \leq U$. Choose an integer $M$, a positive constant $C$ and a constant $\lambda>1$ such that for all $n \geq M$ the following inequalities hold:

$$
r_{n}=\left|x_{n}\right|, \quad \frac{\left|x_{n+1}\right|}{\left|x_{n}\right|+U} \geq \lambda, \quad \prod_{k=0}^{n}\left(1-\frac{\left|x_{k}\right|}{\left|x_{n}\right|}\right) \geq \frac{1}{C} .
$$

For $n \geq M$ we then have

$$
\left|\phi_{X, n}(z)\right| \leq \frac{C\left|z-x_{0}\right| \cdot\left|z-x_{1}\right| \ldots\left|z-x_{n-1}\right|}{\left|x_{n}\right|^{n}} \leq \frac{C}{\lambda^{n}} .
$$

So the series converges uniformly for $|z| \leq U$, and hence determines an entire function in the disc of radius $U$ (see for example $[19, \S 2.8]$ ). Since $U$ was taken arbitrarily, this proves the proposition.

Let $X$ be a parade and suppose that the envelope function $T_{X}$ is an entire function. Informally, the notion of granularity for $X$, defined below, registers the property that for large $n$ there is a radius $r>x_{n}$ at which $\phi_{X, n}$ represents essentially all the mass of $T_{X}$. The precise formulation is tailored to the employment of this notion in the proofs of our main theorems in the next section. For an entire function $f$ we let $m(f, r)$ denote the minimum of $|f(z)|$ for $|z|=r$. We will say that $X$ is granular if

$$
\lim _{n \rightarrow \infty} \inf _{r \geq r_{n}} \frac{r}{r-r_{n}} \cdot \frac{M\left(T_{X}, r\right)}{m\left(\phi_{X, n}, r\right)}=1
$$

We will say that $X$ is semigranular if

$$
\inf _{r \geq r_{n}} \frac{r}{r-r_{n}} \cdot \frac{M\left(T_{X}, r\right)}{m\left(\phi_{X, n}, r\right)}
$$

has an upper bound independent of $n$.

Proposition 3.6. Let $X$ be a diffuse parade. Then $T_{X}$ is granular.

Proof. Write $X=\left\{x_{0}, x_{1}, \ldots\right\}$. Since $X$ is diffuse we may, by Lemma 3.4 , choose a non-negative integer $M$ such that, for $k \geq M$, we have $r_{k}=\left|x_{k}\right|$ and

$$
\prod_{j=0}^{k-1}\left(1+\frac{\left|x_{j}\right|}{\left|x_{k}\right|}\right) \leq 2, \quad \prod_{j=0}^{k-1}\left(1-\frac{\left|x_{j}\right|}{\left|x_{k}\right|}\right) \geq \frac{1}{2} .
$$

For each integer $n$ we set $S=S_{n}=r_{n}^{n}$ and choose $N=N_{n}$ such that $r_{N} \leq S \leq r_{N+1}$. We also set

$$
q=q_{n}=\max _{k \geq n} \frac{\left|x_{k}\right|^{2 k}}{\left|x_{k+1}\right|^{k+1}}, \quad h=h_{n}=\max _{k \geq n} \frac{\left|x_{k}\right|}{\left|x_{k+1}\right|} .
$$

It follows from the diffusity that $\lim _{n \rightarrow \infty} q_{n}=\lim _{n \rightarrow \infty} h_{n}=0$. 
We will show that

$$
\lim _{n \rightarrow \infty} \frac{S}{S-r_{n}} \cdot \frac{M\left(T_{X}, S\right)}{m\left(\phi_{X, n}, S\right)}=1 .
$$

That $S /\left(S-r_{n}\right) \rightarrow 1$ as $n \rightarrow \infty$ is clear; we prove the same for the second factor.

We have

$$
1 \leq \frac{M\left(\phi_{X, n}, S\right)}{m\left(\phi_{X, n}, S\right)} \leq \frac{\prod_{k=0}^{n-1}\left(1+\left|x_{k}\right| /\left|x_{n}\right|\right)}{\prod_{k=0}^{n-1}\left(1-\left|x_{k}\right| /\left|x_{n}\right|\right)} .
$$

It follows by Lemma 3.3 that

$$
\lim _{n \rightarrow \infty} \frac{M\left(\phi_{X, n}, S\right)}{m\left(\phi_{X, n}, S\right)}=1
$$

We set

$$
T_{n}^{*}=T_{X, n}^{*}=T_{X}-\phi_{X, n} .
$$

To establish the proposition it suffices to prove that

$$
\lim _{n \rightarrow \infty} \frac{M\left(T_{n}^{*}, S_{n}\right)}{m\left(\phi_{X, n}, S_{n}\right)}=0
$$

We have

$$
M\left(T_{n}^{*}, S\right) \leq \sum_{k=0}^{n-1} M\left(\phi_{X, k}, S\right)+\sum_{k=n+1}^{\infty} M\left(\phi_{X, k}, S\right) .
$$

We split the sums on the right hand side into six sums $\Sigma_{1}, \Sigma_{2}, \ldots, \Sigma_{6}$ with the following ranges:

$$
\begin{array}{r}
k \leq M-1, \quad M \leq k \leq n-2, \quad k=n-1, \quad n+1 \leq k \leq N, \\
k=N+1 \text { and } k \geq N+2 .
\end{array}
$$

For any positive $r$ and non-negative integer $n$ we have, since the differences $\left|x_{i}-x_{j}\right|$ are all integers,

$$
M\left(\phi_{X, k}, r\right) \leq \frac{\left(r+\left|x_{0}\right|\right)\left(r+\left|x_{1}\right|\right) \ldots\left(r+\left|x_{k-1}\right|\right)}{\left|x_{k}-x_{0}\right| \cdot\left|x_{k}-x_{1}\right| \ldots\left|x_{k}-x_{k-1}\right|} \leq\left(2 \max \left\{r, r_{k-1}\right\}\right)^{k} .
$$

However, this estimate may be improved in various circumstances. If $k \geq M$ then, by our assumption on $M$, we can improve this estimate by observing that

$$
\left|x_{k}-x_{0}\right| \cdot\left|x_{k}-x_{1}\right| \ldots\left|x_{k}-x_{k-1}\right| \geq \frac{1}{2}\left|x_{k}\right|^{k},
$$

while if $k \geq M$ and $r \geq\left|x_{k}\right|$ then

$$
\left(r+\left|x_{0}\right|\right)\left(r+\left|x_{1}\right|\right) \ldots\left(r+\left|x_{k-1}\right|\right) \leq 2 r^{k} .
$$


The first range is estimated crudely by

$$
\Sigma_{1}=\sum_{k=0}^{M-1} M\left(\phi_{X, k}, S\right) \leq M(2 S)^{M-1} .
$$

In the second range, where $M \leq k \leq n-2$, we have

$$
\Sigma_{2}=\sum_{k=M}^{n-2} M\left(\phi_{X, k}, S\right) \leq \sum_{k=M}^{n-2} 2 S^{k} \leq 2 n S^{n-2} .
$$

In the third range $k=n-1$ so that

$$
\Sigma_{3}=M\left(\phi_{X, n-1}, S\right) \leq 4 S^{n-1} / r_{n-1}^{n-1} .
$$

To estimate the sum over the fourth range $n+1 \leq k \leq N$, we observe that, for $j$ non-negative,

$$
\frac{S}{r_{n+j+1}^{n+j+1}}=\frac{\left(r_{n+j}^{n+j}\right)^{2}}{r_{n+j+1}^{n+j+1}}\left(\frac{S}{r_{n+j}^{n+j}}\right)^{2} \frac{1}{S} \leq q\left(\frac{S}{r_{n+j}^{n+j}}\right)^{2} .
$$

Since $S / r_{n+1}^{n+1} \leq q$ it follows that $S / r_{n+j}^{n+j} \leq q^{2^{j}-1}$. Thus

$$
\frac{S^{n+j+1}}{r_{n+j+1}^{n+j+1}} \cdot \frac{r_{n+j}^{n+j}}{S^{n+j}}=\frac{\left(r_{n+j}^{n+j}\right)^{2}}{r_{n+j+1}^{n+j+1}} \cdot \frac{S}{r_{n+j}^{n+j}} \leq q q^{2^{j}-1} \leq q^{2^{j}} .
$$

Hence

$$
\Sigma_{4}=\sum_{k=n+1}^{N} M\left(\phi_{X, k}, S\right) \leq 4 \sum_{j=0}^{\infty} \frac{S^{n+j+1}}{r_{n+j+1}^{n+j+1}} \leq 4 \frac{S^{n}}{r_{n}^{n}} \sum_{j=0}^{\infty} q^{2^{j+1}-1} \leq \frac{4 q}{1-q} \cdot \frac{S^{n}}{r_{n}^{n}} .
$$

The fifth range is estimated by observing that

$$
\left(S+\left|x_{0}\right|\right)\left(S+\left|x_{1}\right|\right) \ldots\left(S+\left|x_{N-1}\right|\right)\left(S+\left|x_{N}\right|\right) \leq 2 S^{N} 2 S
$$

while

Therefore

$$
\left|x_{N+1}-x_{0}\right| \ldots\left|x_{N+1}-x_{N}\right| \geq \frac{1}{2} r_{N+1}^{N+1} \geq \frac{1}{2} S^{N+1} .
$$

$$
\Sigma_{5}=M\left(\phi_{X, N+1}, S\right) \leq 8 .
$$

In the last range we have $k \geq N+2$ so that $S \leq r_{k-1}$. We have

$$
\prod_{k=N+2}^{\infty}\left(1+\frac{S}{\left|x_{k}\right|}\right) \leq \exp \left(\sum_{j=1}^{\infty} h^{j}\right) \leq \exp \left(\frac{h}{1-h}\right) .
$$

Therefore

$$
\begin{aligned}
\left(S+\left|x_{0}\right|\right) & \ldots\left(S+\left|x_{N}\right|\right)\left(S+\left|x_{N+1}\right|\right)\left(S+\left|x_{N+2}\right|\right) \ldots\left(S+\left|x_{k-1}\right|\right) \\
& \leq 2 \exp \left(\frac{h}{1-h}\right) S^{N+1}\left(2\left|x_{N+1}\right|\right)\left|x_{N+2}\right| \cdot\left|x_{N+3}\right| \ldots\left|x_{k-1}\right|,
\end{aligned}
$$


and so

$$
\begin{aligned}
M\left(\phi_{X, k}, S\right) & \leq 8 \exp \left(\frac{h}{1-h}\right) \frac{S^{N+1}\left|x_{N+1}\right| r_{N+2} \ldots r_{k-1}}{r_{k}^{k}} \\
& \leq 8 h^{k} \exp \left(\frac{h}{1-h}\right) .
\end{aligned}
$$

Thus

$$
\Sigma_{6}=\sum_{k=N+2}^{\infty} M\left(\phi_{X, k}, S\right) \leq 8 \exp \left(\frac{h}{1-h}\right) \sum_{k=n+2}^{\infty} h^{k} \leq \frac{8 h}{1-h} \exp \left(\frac{h}{1-h}\right) .
$$

Now

$$
\begin{aligned}
m\left(\phi_{X, n}, S\right) & \geq \frac{\left(r_{n+1}-\left|x_{0}\right|\right)\left(r_{n+1}-\left|x_{1}\right|\right) \ldots\left(r_{n+1}-\left|x_{n-1}\right|\right)}{\left|x_{n}-x_{0}\right| \cdot\left|x_{n}-x_{1}\right| \ldots\left|x_{n}-x_{n-1}\right|} \\
& \geq \frac{S^{n}}{4 r_{n}^{n}}=\frac{S^{n-1}}{4} .
\end{aligned}
$$

Since $X$ is diffuse we have $q_{n}, h_{n} \rightarrow 0$ as $n \rightarrow \infty$. We have also $S=r_{n}^{n} \geq 2^{n}$ for all sufficiently large $n$. Therefore the sums $\Sigma_{1}, \Sigma_{2}, \Sigma_{3}, \Sigma_{4}, \Sigma_{5}, \Sigma_{6}$ are all $o\left(S^{n-1}\right)$ as $n \rightarrow \infty$.

REMARK 3.7. Under the conditions of Proposition 3.6 we have $M\left(T_{X}, r_{n}^{n}\right) / r_{n}^{n^{2}-n} \rightarrow 1$ as $n \rightarrow \infty$.

For a sequence $X$ of complex numbers we define

$$
R_{n}(X)=\sqrt{\frac{r_{n+1}^{n+1}(X)}{r_{n-1}^{n-1}(X)}} .
$$

Proposition 3.8. Let $X$ be a semidiffuse parade. Then $T_{X}$ is semigranular.

Proof. We set $R_{n}=R_{n}(X)$ and establish an upper bound independent of $n$ for

$$
\frac{R_{n}}{R_{n}-r_{n}} \cdot \frac{M\left(T_{X}, R_{n}\right)}{m\left(\phi_{X, n}, R_{n}\right)}
$$

Such a bound obtains for the first factor by the semidiffusity of $X$. Indeed as $n \rightarrow \infty$ we have

$$
\frac{r_{n}}{R_{n}}=\frac{r_{n} r_{n-1}^{n-1}}{\sqrt{r_{n+1}^{n+1} r_{n-1}^{n-1}}}=\left(\frac{r_{n-1}}{r_{n}}\right)^{n-1} \frac{r_{n}^{n}}{\sqrt{r_{n+1}^{n+1} r_{n-1}^{n-1}}} \rightarrow 0 .
$$

By Lemma 3.5 there is a non-negative integer $M$ and a positive constant $C$ such that, for all $n \geq M$, we have

$$
\prod_{k=0}^{n-1}\left(1+\frac{\left|x_{k}\right|}{\left|x_{n}\right|}\right)<C, \quad \prod_{k=0}^{n-1}\left(1-\frac{\left|x_{k}\right|}{\left|x_{n}\right|}\right)>\frac{1}{C} \text {. }
$$


By the semidiffusity we can assume that there are constants $\lambda>1$ and $\delta>1$ such that (increasing $M$ if necessary)

$$
\frac{\left|x_{n+1}\right|}{\left|x_{n}\right|} \geq \lambda, \quad \frac{\left|x_{n+1}\right|^{n+1}\left|x_{n-1}\right|^{n-1}}{\left|x_{n}\right|^{2 n}} \geq \delta
$$

also hold for all $n \geq M$, and further that $r_{n}=\left|x_{n}\right|$ for all $n \geq M$.

Thus for $n \geq M$ we have

$$
m\left(\phi_{X, n}, R_{n}\right) \geq C^{2} R_{n}^{n} / r_{n}^{n}
$$

Now suppose that $n$ is so large that

$$
\delta^{(n-M-1)(n-M-2) / 2} \geq M\left(2 r_{M}\right)^{M} .
$$

We begin with the estimate

$$
M\left(T_{X}, R_{n}\right) \leq \sum_{k=0}^{\infty} M\left(\phi_{X, k}, R_{n}\right)
$$

and split the sum on the right into three sums over the ranges

$$
k \leq M-1, \quad M \leq k \leq n, \quad k>n .
$$

To estimate the sum over the range $k>n$ we note that, for $j$ nonnegative,

$$
\begin{aligned}
\frac{R_{n} r_{n+j}^{n+j}}{r_{n+j+1}^{n+j+1}} & =\frac{r_{n+j}^{2(n+j)}}{r_{n+j+1}^{n+j+1} r_{n+j-1}^{n+j-1}} \cdot \frac{R_{n} r_{n+j-1}^{n+j-1}}{r_{n+j}^{n+j}} \leq \delta^{-1} \frac{R_{n} r_{n+j-1}^{n+j-1}}{r_{n+j}^{n+j}} \\
& \leq \ldots \leq \delta^{-j} \frac{R_{n} r_{n}^{n}}{r_{n+1}^{n+1}} \leq \delta^{-j} .
\end{aligned}
$$

Therefore

$$
\frac{R_{n}^{n+j+1}}{r_{n+j+1}^{n+j+1}} \cdot \frac{r_{n}^{n}}{R_{n}^{n}} \leq \delta^{-j(j-1) / 2},
$$

so that

$$
\sum_{k=n+1}^{\infty} M\left(\phi_{X, k}, R_{n}\right) \leq \frac{R_{n}^{n}}{r_{n}^{n}} \sum_{j=1}^{\infty} \delta^{-j(j-1) / 2} \leq \frac{R_{n}^{n}}{r_{n}^{n}} \cdot \frac{\delta}{\delta-1} .
$$

For the sum over the second range we proceed similarly, noting that for non-negative $j$ with $n-j \geq M$ we have

$$
\begin{aligned}
\frac{r_{n-j}^{n-j}}{R_{n} r_{n-j-1}^{n-j-1}} & =\frac{r_{n-j}^{2(n-j)}}{r_{n-j-1}^{n-j-1} r_{n-j+1}^{n-j+1}} \cdot \frac{r_{n-j+1}^{n-j+1}}{R_{n} r_{n-j}^{n-j}} \leq \delta^{-1} \frac{r_{n-j+1}^{n-j+1}}{R_{n} r_{n-j}^{n-j}} \\
& \leq \ldots \leq \delta^{-j} \frac{R_{n} r_{n}^{n}}{r_{n-1}^{n-1}} \leq \delta^{-j},
\end{aligned}
$$


so that

$$
\frac{R_{n}^{n-j-1}}{r_{n-j-1}^{n-j-1}} \cdot \frac{r_{n}^{n}}{R_{n}^{n}} \leq \delta^{-j(j-1) / 2},
$$

yielding

$$
\sum_{k=M}^{n-1} M\left(\phi_{X, k}, R_{n}\right) \leq \frac{R_{n}^{n}}{r_{n}^{n}} \sum_{j=1}^{\infty} \delta^{-j(j-1) / 2} \leq \frac{R_{n}^{n}}{r_{n}^{n}} \cdot \frac{\delta}{\delta-1} .
$$

For the range $k \leq M-1$ we have

$$
\sum_{k=0}^{M-1} M\left(\phi_{X, k}, R_{n}\right) \leq M\left(2 R_{n}\right)^{M} .
$$

From the discussion of the second range it follows that

$$
\frac{R_{n}^{M}}{r_{M}^{M}} \cdot \frac{r_{n}^{n}}{R_{n}^{n}} \leq \delta^{-(n-M-1)(n-M-2) / 2} .
$$

Hence

$$
\frac{\sum_{k=0}^{M-1} M\left(\phi_{X, k}, R_{n}\right)}{m\left(\phi_{X, n}, R_{n}\right)} \leq C^{2} \frac{M\left(2 r_{M}\right)^{M}}{\delta^{(n-M-1)(n-M-2) / 2}} \leq C^{2} .
$$

Thus

$$
\frac{M\left(T_{X}, R_{n}\right)}{m\left(\phi_{X, n}, R_{n}\right)} \leq C^{2}\left(1+\frac{2 \delta}{\delta-1}\right)
$$

and this completes the proof.

Corollary 3.9. Let $X$ be a semidiffuse parade. Then $M\left(T_{X}, R_{n}\right) r_{n}^{n} / R_{n}^{n}$ is bounded independently of $n$.

Pr o of. This follows from the proof of Proposition 3.8.

4. Proof of Theorem 1.2. In this section we prove Theorem 1.2. In fact we prove the following.

Theorem 4.1. Let $X$ be a parade with $T_{X}$ entire. Suppose that $T_{X}$ is granular. Let $f$ be an entire function that takes integral values on $X$ and satisfies

$$
\limsup _{r \rightarrow \infty} \frac{M(f, r)}{M\left(T_{X}, r\right)}<1 .
$$

Then $f$ is a polynomial.

We also prove the following version that, in view of Proposition 3.3, applies to semidiffuse parades. However, we do not know of any examples of parades that are semidiffuse but not diffuse that are essentially different from the case treated by Gel'fond; that is, sequences generated by iteration of a linear polynomial with non-unit slope. 
Theorem 4.2. Let $X$ be a parade with $T_{X}$ entire. Suppose that $T_{X}$ is semigranular. Let $f$ be an entire function that takes integral values on $X$ and satisfies

$$
\limsup _{r \rightarrow \infty} \frac{M(f, r)}{M\left(T_{X}, r\right)}=0 .
$$

Then $f$ is a polynomial.

Suppose that $f$ is an entire $X$-integral function in the hypothesis of Theorem 4.1 or 4.2 . The strategy of the proof of Theorems 4.1 and 4.2, following the strategies of Pólya [15, 16], is as follows. First, construct a polynomial $Q$ that takes the same values as $f$ on $X$. Second, prove that $f$ and $Q$ are identically equal. The approach used by Gel'fond [8, 9] and Bézivin $[1,2]$ is slightly different. They show that $f$ can be represented by an interpolation series with respect to $X$, and then show that this series terminates after finitely many terms so that it is a polynomial.

We begin with four propositions. The first three evaluate the coefficients that will be used to construct $Q$ out of the polynomials $\phi_{X, n}$. The fourth gives the requisite uniqueness of interpolation and is analogous to a Hilfssatz $[15, \S 4]$ of Pólya.

We denote by $V\left(x_{0}, \ldots, x_{n}\right)$ the Vandermonde determinant on $x_{0}, \ldots$ $\ldots, x_{n}$. If $X=\left\{x_{0}, x_{1}, \ldots\right\}$ is a sequence of complex numbers containing at least $n+1$ elements we set $V_{n}(X)=V\left(x_{0}, x_{1}, \ldots, x_{n}\right)$.

Proposition 4.3. Let $\left\{x_{0}, x_{1}, \ldots, x_{n}\right\} \subset \mathbb{C}$ be a set of distinct points and let $\psi_{j}, j=0,1, \ldots, n$, and $f$ be functions defined on $\left\{x_{0}, x_{1}, \ldots, x_{n}\right\}$. Suppose that $\psi_{j}\left(x_{j}\right)=1, j=0,1, \ldots, n$, and $\psi_{j}\left(x_{i}\right)=0$ whenever $i<j$. For $m=0,1, \ldots, n$ set

$$
c_{m}=\operatorname{det}\left(\begin{array}{ccccc}
\psi_{0}\left(x_{0}\right) & \psi_{1}\left(x_{0}\right) & \ldots & \psi_{m-1}\left(x_{0}\right) & f\left(x_{0}\right) \\
\vdots & \vdots & & \vdots & \vdots \\
\psi_{0}\left(x_{m}\right) & \psi_{1}\left(x_{m}\right) & \ldots & \psi_{m-1}\left(x_{m}\right) & f\left(x_{m}\right)
\end{array}\right) \text {. }
$$

Then

$$
f\left(x_{k}\right)=\sum_{j=0}^{n} c_{j} \psi_{j}\left(x_{k}\right), \quad k=0,1, \ldots, n .
$$

Proof. The proof is by induction: for $n=0$, the conclusion holds because $c_{0}=f\left(x_{0}\right)$ and $\psi_{0}\left(x_{0}\right)=1$.

Assuming that the conclusion holds for $n$ points, we prove it for $n+1$. Since $\psi_{n}\left(x_{k}\right)=0$ for $k=0, \ldots, n-1$, the induction hypothesis shows that

$$
f\left(x_{k}\right)=\sum_{j=0}^{n} c_{j} \psi_{j}\left(x_{k}\right), \quad k=0,1, \ldots, n-1 .
$$


So to prove the proposition we need only check that

$$
f\left(x_{n}\right)=\sum_{j=0}^{n} c_{j} \psi_{j}\left(x_{n}\right) .
$$

In the determinantal expression for $c_{n}$, we subtract from the last column a linear combination of the previous columns to obtain

$$
c_{n}=\operatorname{det}\left(\begin{array}{ccccc}
\psi_{0}\left(x_{0}\right) & \psi_{1}\left(x_{0}\right) & \ldots & \psi_{n-1}\left(x_{0}\right) & f\left(x_{0}\right)-\sum_{j=0}^{n-1} c_{j} \psi_{j}\left(x_{0}\right) \\
\vdots & \vdots & & \vdots & \vdots \\
\psi_{0}\left(x_{n}\right) & \psi_{1}\left(x_{n}\right) & \ldots & \psi_{n-1}\left(x_{n}\right) & f\left(x_{n}\right)-\sum_{j=0}^{n-1} c_{j} \psi_{j}\left(x_{n}\right)
\end{array}\right)
$$

In view of the induction hypothesis, this matrix is lower triangular. The diagonal terms, apart from the $(n+1, n+1)$ term, are equal to 1 . Hence

$$
c_{n}=f\left(x_{n}\right)-\sum_{j=0}^{n-1} c_{j} \psi_{j}\left(x_{n}\right) \text {. }
$$

Since $\psi_{n}\left(x_{n}\right)=1$ we have

$$
\sum_{j=0}^{n} c_{j} \psi_{j}\left(x_{n}\right)=\sum_{j=0}^{n-1} c_{j} \psi_{j}\left(x_{n}\right)+c_{n} \psi_{n}\left(x_{n}\right)=f\left(x_{n}\right),
$$

completing the proof.

Proposition 4.4. Let $x_{0}, x_{1}, \ldots, x_{n}, z$ be independent indeterminates. Then

$$
\operatorname{det}\left(\begin{array}{ccccc}
1 & x_{0} & \ldots & x_{0}^{n-1} & \frac{1}{z-x_{0}} \\
1 & x_{1} & \ldots & x_{1}^{n-1} & \frac{1}{z-x_{1}} \\
\vdots & \vdots & & \vdots & \vdots \\
1 & x_{n} & \ldots & x_{n}^{n-1} & \frac{1}{z-x_{n}}
\end{array}\right)=\frac{V\left(x_{0}, \ldots, x_{n}\right)}{\left(z-x_{0}\right)\left(z-x_{1}\right) \ldots\left(z-x_{n}\right)} .
$$

P r o of. Multiplying the last column by $P=\left(z-x_{0}\right)\left(z-x_{1}\right) \ldots\left(z-x_{n}\right)$ we find that

$$
\begin{aligned}
\operatorname{det}\left(\begin{array}{ccccc}
1 & x_{0} & \ldots & x_{0}^{n-1} & \frac{1}{z-x_{0}} \\
1 & x_{1} & \ldots & x_{1}^{n-1} & \frac{1}{z-x_{1}} \\
\vdots & \vdots & & \vdots & \vdots \\
1 & x_{n} & \ldots & x_{n}^{n-1} & \frac{1}{z-x_{n}}
\end{array}\right) \\
\quad=\frac{1}{P} \operatorname{det}\left(\begin{array}{ccccc}
1 & x_{0} & \ldots & x_{0}^{n-1} & \left(\widetilde{z-x_{0}}\right)\left(z-x_{1}\right) \ldots\left(z-x_{n}\right) \\
1 & x_{1} & \ldots & x_{1}^{n-1} & \left(z-x_{0}\right)\left(z-x_{1}\right) \ldots\left(z-x_{n}\right) \\
\vdots & \vdots & & \vdots & \vdots \\
1 & x_{n} & \ldots & x_{n}^{n-1} & \left(z-x_{0}\right)\left(z-x_{1}\right) \ldots\left(\widehat{z-x_{n}}\right)
\end{array}\right)
\end{aligned}
$$


where ${ }^{\wedge}$ denotes an omitted term. Using column linearity, we can expand the determinant on the right hand side above into a polynomial in $z$ whose coefficients are determinants in the other variables. These coefficient determinants vanish if we have $x_{i}=x_{j}$ for some $i \neq j$, and hence are divisible by $V\left(x_{0}, \ldots, x_{n}\right)$. Therefore, considering degrees, all these coefficients for positive powers of $z$ vanish. The coefficient of $z^{0}$ is some constant multiple of $V\left(x_{0}, \ldots, x_{n}\right)$, and this constant can be seen to be 1 by considering the case $x_{i}=i$.

Given a proper infinite sequence $X$ of complex numbers and a complexvalued function $f$ defined on $X$ we set, for each non-negative integer $n$,

$$
c_{n}(X, f)=\operatorname{det}\left(\begin{array}{ccccc}
\phi_{X, 0}\left(x_{0}\right) & \phi_{X, 1}\left(x_{0}\right) & \ldots & \phi_{X, n-1}\left(x_{0}\right) & f\left(x_{0}\right) \\
\vdots & \vdots & & \vdots & \vdots \\
\phi_{X, 0}\left(x_{n}\right) & \phi_{X, 1}\left(x_{n}\right) & \ldots & \phi_{X, n-1}\left(x_{n}\right) & f\left(x_{n}\right)
\end{array}\right) .
$$

Using

$$
\phi_{X, n}(x)=\frac{\left(x-x_{0}\right)\left(x-x_{1}\right) \ldots\left(x-x_{n-1}\right)}{\left(x_{n}-x_{0}\right)\left(x_{n}-x_{1}\right) \ldots\left(x_{n}-x_{n-1}\right)}
$$

and elementary column operations we get the alternative expression

$$
c_{n}(X, f)=\frac{1}{V_{n-1}(X)} \operatorname{det}\left(\begin{array}{ccccc}
1 & x_{0} & \ldots & x_{0}^{n-1} & f\left(x_{0}\right) \\
1 & x_{1} & \ldots & x_{1}^{n-1} & f\left(x_{1}\right) \\
\vdots & \vdots & & \vdots & \vdots \\
1 & x_{n} & \ldots & x_{n}^{n-1} & f\left(x_{n}\right)
\end{array}\right) .
$$

Proposition 4.5. Let $X=\left\{x_{0}, x_{1}, \ldots\right\} \subset \mathbb{C}$ be a proper infinite sequence and $n$ a non-negative integer. Let $r>\max \left\{\left|x_{0}\right|, \ldots,\left|x_{n}\right|\right\}$. Let $D_{r}$ denote the open disk of radius $r$ centered at the origin in the complex plane, and $C_{r}$ its boundary, and suppose that $f$ is holomorphic in a neighborhood of $D_{r} \cup C_{r}$. Then

$$
c_{n}(X, f)=\frac{V_{n}(X)}{2 \pi i V_{n-1}(X)} \int_{C_{r}} \frac{f(z)}{\left(z-x_{0}\right)\left(z-x_{1}\right) \ldots\left(z-x_{n}\right)} d z .
$$

Proof. Applying Cauchy's integral theorem to the second expression above for $c_{n}(X, f)$ we find

$$
c_{n}(X, f)=\frac{1}{2 \pi i V_{n-1}(X)} \int_{C_{r}} f(z) \operatorname{det}\left(\begin{array}{ccccc}
1 & x_{0} & \ldots & x_{0}^{n-1} & \frac{1}{z-x_{0}} \\
1 & x_{1} & \ldots & x_{1}^{n-1} & \frac{1}{z-x_{1}} \\
\vdots & \vdots & & \vdots & \vdots \\
1 & x_{n} & \ldots & x_{n}^{n-1} & \frac{1}{z-x_{n}}
\end{array}\right) d z .
$$

The conclusion now follows by Proposition 4.3. 
Proposition 4.6. Let $X$ be a semidiffuse sequence of complex numbers and define $R_{n}=R_{n}(X)$ as in Section 3. Suppose that

$$
1 \leq \lambda<\liminf _{n \rightarrow \infty} \frac{\left|x_{n+1}\right|}{\left|x_{n}\right|}
$$

Let $f$ be an entire function with

$$
\lim _{n \rightarrow \infty} \frac{M\left(f, R_{n}\right)}{\lambda^{n(n-1) / 2} M\left(T_{X}, R_{n}\right)}=0
$$

Then the interpolation series $\sum_{n=0}^{\infty} c_{n}(X, f) \phi_{X, n}(z)$ is entire and equal to $f$.

Proof. Let $t, z, y_{0}, y_{1}, \ldots$ be independent indeterminates, and define the polynomials $P_{0}(z)=1, P_{1}(z)=\left(z-y_{0}\right), \ldots, P_{k}(z)=\left(z-y_{0}\right)\left(z-y_{1}\right) \ldots(z-$ $\left.y_{k-1}\right), \ldots$ It is easily shown by induction (or see [9]) that, for each $n$,

$$
\frac{1}{t-z}=\frac{P_{0}(z)}{P_{1}(t)}+\frac{P_{1}(z)}{P_{2}(t)}+\ldots+\frac{P_{n-1}(z)}{P_{n}(t)}+\frac{P_{n}(z)}{(t-z) P_{n}(t)} .
$$

If now $z$ is complex and $r>\max \left\{|z|,\left|x_{i}\right| \mid i=0, \ldots, n\right\}$ then the above identity and the formula for $c_{n}(X, f)$ of Proposition 4.5 yields

$$
f(z)=\frac{1}{2 \pi i} \int_{C_{r}} \frac{f(t)}{t-z} d t=\sum_{k=1}^{n} c_{k}(X, f) \phi_{X, k}(z)+W_{n}(z)
$$

where

$$
W_{n}(z)=P_{n}(z) \frac{1}{2 \pi i} \int_{C-r} \frac{f(t)}{(t-z) P_{n}(t)} d t=\phi_{X, n}(z) \frac{1}{2 \pi i} \int_{C-r} \frac{f(t)}{(t-z) \phi_{X, n}(t)} d t
$$

Taking $r=R_{n}$ we have, once $r_{n}>|z|$,

$$
\left|W_{n}(z)\right| \leq\left|\phi_{X, n}(z)\right| \frac{R_{n} M\left(f, R_{n}\right)}{\left(R_{n}-r_{n}\right) m\left(\phi_{X, n}, R_{n}\right)} .
$$

Since $X$ is semidiffuse, $T_{X}$ is semigranular so that, under the growth hypothesis on $f$,

$$
\left|W_{n}(z)\right| \leq \lambda^{n(n-1) / 2}\left|\phi_{X, n}(z)\right|
$$

once $n$ is large enough. Choose $\mu>\lambda$ such that, for all sufficiently large $m$ (say $m>M$ ), we have

$$
\frac{\left|x_{m+1}\right|}{\left|x_{m}\right|+|z|} \geq \mu .
$$

Then there is a positive constant $H$ such that

$$
\left|\phi_{X, n}(z)\right| \leq H \mu^{-(n-M)(n-M-1) / 2}
$$

so that $W_{n}(z) \rightarrow 0$ as $n \rightarrow \infty$. 
COROLlary 4.7. Let the entire function $f$ satisfy the growth hypothesis of Proposition 4.6 and suppose that $f$ vanishes on $X$. Then $f$ vanishes identically.

REMARK. Corollary 4.7 may be proved directly from Jensen's Theorem in a straightforward way, analogous to a lemma of Pólya [15] showing that an entire function $f$ vanishing on $\mathbb{N}$ and satisfying $M(f, r) \leq \beta^{r}$ for some $\beta<e$ vanishes identically. Carlson's theorem (see $[19,5.81]$ ) improves this, showing that the same conclusion holds if $f$ vanishes on $\mathbb{N}$ and satisfies $M(f, r) \leq \beta^{r}$ for some $\beta<e^{\pi}$. This is essentially best possible in view of the function $\sin (\pi z)$. It would be interesting to try to improve Corollary 4.7 in an analogous way.

Proof of Theorems 4.1 and 4.2. By Proposition 4.6 we have

$$
f(z)=\sum_{j=0}^{\infty} c_{j}(X, f) \phi_{X, j}(z) .
$$

We need only show that the $c_{n}(X, f)$ vanish for all sufficiently large $n$.

Since all the entries in the matrix determining $c_{n}(X, f)$ are integers it follows that each $c_{n}(X, f)$ is an integer. We proceed to estimate $c_{n}(X, f)$. Since

Set $r_{n}=r_{n}(X)=\max \left\{\left|x_{0}\right|,\left|x_{1}\right|, \ldots,\left|x_{n}\right|\right\}$ as in Section 3 and let $r>r_{n}$.

$$
\frac{V_{n}(X)}{V_{n-1}(X)\left(r-x_{0}\right)\left(r-x_{1}\right) \ldots\left(r-x_{n}\right)}=\frac{1}{\left(r-x_{n}\right) \phi_{X, n}(r)},
$$

the expression for $c_{n}(X, f)$ of Proposition 4.5 yields the estimate

$$
\left|c_{n}(X, f)\right| \leq \frac{r}{r-\left|x_{n}\right|} \cdot \frac{M(f, r)}{m\left(\phi_{X, n}, r\right)} .
$$

Hence

$$
\left|c_{n}(X, f)\right| \leq \inf _{r>r_{n}}\left(\frac{r}{r-r_{n}} \cdot \frac{M(f, r)}{m\left(\phi_{X, n}, r\right)}\right)
$$

which we rewrite as

$$
\left|c_{n}(X, f)\right| \leq \inf _{r>r_{n}}\left(\frac{r}{r-r_{n}} \cdot \frac{M\left(T_{X}, r\right)}{m\left(\phi_{X, n}, r\right)} \cdot \frac{M(f, r)}{M\left(T_{X}, r\right)}\right) .
$$

Under the hypotheses of Theorem 4.1 we have

$$
\limsup _{n \rightarrow \infty}\left|c_{n}(X, f)\right| \leq \limsup _{r \rightarrow \infty} \frac{M(f, r)}{M\left(T_{X}, r\right)}<1 .
$$

Thus there is an integer $N$ such that $\left|c_{n}(X, f)\right|<1$ for all $n>N$, and since $c_{n}(X, f)$ is an integer we in fact have $c_{n}(X, f)=0$ for all $n>N$. 
Under the hypotheses of Theorem 4.2 we have

$$
\inf _{r>r_{n}}\left(\frac{r}{r-r_{n}} \cdot \frac{M\left(T_{X}, r\right)}{m\left(\phi_{X, n}, r\right)}\right)
$$

bounded independently of $n$ while

$$
\lim _{r \rightarrow \infty} \frac{M(f, r)}{M\left(T_{X}, r\right)}=0 .
$$

Hence again we find that there is an integer $N$ such that $\left|c_{n}(X, f)\right|<1$ for all $n>N$ and thus $c_{n}(X, f)=0$ for all $n>N$.

Proof of Theorem 1.2. Suppose that $X$ is diffuse. Then $T_{X}$ is granular by Proposition 3.6, and the conclusion of Theorem 1.2 follows from Theorem 4.1 .

5. Processions. Let $X$ be a parade. We call $X$ a procession if $0 \leq x_{0}<$ $x_{1}<\ldots$

If $X=\left\{x_{0}, x_{1}, \ldots\right\}$ is a parade and $Y=\left\{y_{0}, y_{1}, \ldots\right\}$ is a procession then we can form a new sequence denoted $X_{Y}$ by setting

$$
X_{Y}=Z=\left\{z_{0}, z_{1}, \ldots\right\}, \quad z_{n}=x_{y_{n}}, \quad n=0,1, \ldots
$$

If $X$ is a procession then clearly $X_{Y}$ is also a procession.

Proposition 1.1 may be restated as follows.

Proposition 5.1. Let $X$ be a concordant parade and $Y$ a concordant procession. Then $X_{Y}$ is a concordant parade.

Pro of. Let $z_{0}, z_{1}, \ldots$ denote the elements of $X_{Y}$, so that $z_{i}=x_{y_{i}}$. Fix $d$ and consider the sequence of residues of $x_{0}, x_{1}, \ldots$ in $\mathbb{Z} / d \mathbb{Z}$. Let $h, h+k$ be the smallest two indices with $x_{h} \equiv x_{h+k} \bmod d$. Then, for any indices $s, t$ with, say, $s<t$, we have $x_{s} \equiv x_{t} \bmod d$ if and only if $s \geq h$ and $s \equiv t \bmod k$. Suppose now that $i<j$ and $z_{i} \equiv z_{j} \bmod d$. It follows that $y_{i}, y_{j} \geq h$ and $y_{i} \equiv y_{j} \bmod k$. Since $Y$ is concordant we have $y_{i+1} \equiv y_{j+1} \bmod k$, and since $Y$ is a procession we have $y_{i+1}, y_{j+1} \geq h$; hence $z_{i+1} \equiv z_{j+1} \bmod d$.

Proposition 5.2. Let $X=\left\{x_{0}, x_{1}, \ldots\right\}$ be a diffuse sequence of complex numbers and let $Y=\left\{y_{0}, y_{1}, \ldots\right\}$ be a subsequence of $X$. Then $Y$ is diffuse.

Proof. Define the sequence $A_{n}$ by setting

$$
\left|x_{n+1}\right|^{n+1}=A_{n}\left|x_{n}\right|^{2 n} .
$$

It follows from our hypotheses that $A_{n} \rightarrow \infty$ as $n \rightarrow \infty$. Define similarly the sequence $B_{m}$ by

$$
\left|y_{m+1}\right|^{m+1}=B_{m}\left|y_{m}\right|^{2 m} .
$$

We must show that $B_{m} \rightarrow \infty$ as $m \rightarrow \infty$. 
Take $M$ so large that $\left|x_{n}\right|<\left|x_{n+1}\right|$ for all $n \geq M$. We may suppose that $n>M$

Suppose that $y_{m}=x_{n}$, where $m \leq n$. Then $y_{m+1}=x_{n+j}$ for some $j \geq 1$ so that $\left|y_{m+1}\right| \geq\left|x_{n+1}\right|$. We then have

$$
\begin{aligned}
\left|y_{m+1}\right|^{m+1} & \geq\left|x_{n+1}\right|^{m+1}=\left|A_{n} x_{n}^{2 n}\right|^{(m+1) /(n+1)} \\
& =A_{n}^{(m+1) /(n+1)}\left|y_{m}\right|^{2 m}\left|y_{m}\right|^{2(n-m) /(n+1)} .
\end{aligned}
$$

Thus

$$
B_{m} \geq A_{n}^{(m+1) /(n+1)}\left|y_{m}\right|^{2(n-m) /(n+1)} .
$$

Since we must have either $m \leq n / 2$ or $m>n / 2$ we have (for $n \geq 1$ )

$$
B_{m} \geq \min \left\{\sqrt{A_{n}}, \sqrt{\left|y_{m}\right|}\right\}
$$

so that $B_{m} \rightarrow \infty$ as $m \rightarrow \infty$ as required.

These two results imply that if $X$ is a diffuse concordant sequence and $Y$ is a concordant procession then $X_{Y}$ is a diffuse concordant sequence. The choice $X=X_{a, b}$ and $Y=X_{c}$ yields the example $Z=X_{a, b, c}$ mentioned in the introduction. Setting $W=X_{b, c, d}$ and $X_{a, b, c, d}=X_{W}$ yields the example

$$
X_{a, b, c, d}=\left\{a^{c^{c^{d^{n}}}} \mid n=0,1, \ldots\right\} .
$$

The following proposition deals with sequences of the form $X_{Y}$ when $X$ and $Y$ are generated by linear polynomials with non-unit slope.

Proposition 5.3. Let $P, Q \in \mathbb{Z}[x]$ be linear with non-unit slope. Suppose that $x_{0}, y_{0} \in \mathbb{Z}$. Set $X=X_{P, x_{0}}$ and $Y=X_{Q, y_{0}}$ and suppose that $X$ is proper and that $Y$ is a procession. Then $X_{Y}$ is a diffuse parade.

Proof. Let $X_{Y}=\left\{z_{0}, z_{1}, \ldots\right\}$. Let $a$ be the leading coefficient of $P$ and write $Q(x)=b x+g$. There are constants $H, K, M$ such that, for $n \geq M$,

$$
H|a|^{n-M} \leq\left|x_{n}\right| \leq K|a|^{n-M} \text {. }
$$

Hence

$$
\frac{\left|z_{n+1}\right|^{n+1}}{\left|z_{n}\right|^{2 n}} \geq \frac{\left(H|a|^{y_{n+1}-M}\right)^{n+1}}{\left(K|a|^{y_{n}-M}\right)^{2 n}} \geq \frac{H^{n+1}}{K^{2 n}}|a|^{(n+1) y_{n+1}-2 n y_{n}} .
$$

Now

$$
\begin{aligned}
(n+1) y_{n+1}-2 n y_{n} & =y_{n}\left((n+1) b\left(1+g / y_{n}\right)-2 n\right) \\
& \geq y_{n}\left((n+1) 2\left(1+g / y_{n}\right)-2 n\right) \geq y_{n} / 2
\end{aligned}
$$

for all sufficiently large $n$. Thus $\left|z_{n+1}\right|^{n+1} /\left|z_{n}\right|^{2 n} \rightarrow \infty$ as $n \rightarrow \infty$.

6. Envelope functions. In this section we study the envelope functions $T_{a}$. We show that $T_{a}$ satisfies a recurrence relation, and derive therefrom a combinatorial expression for its Taylor series. The recurrence relation for $T_{a}$ also appears in a paper [6] of Bundschuh; we include a proof for the reader's 
convenience. We thank the referee for making us aware of Bundschuh's paper. It would be interesting to investigate whether analogous recurrence relations are satisfied by other envelope functions.

We denote the Taylor expansion of $T_{X}$ by

$$
T_{X}(z)=\sum_{n=0}^{\infty} t_{n}(X) z^{n} .
$$

For $x$ an indeterminate and $a$ in some valued field with $|a|>1$ let

$$
T(x)=T_{a}(x)=1+\sum_{n=1}^{\infty} \frac{(x-1) \ldots\left(x-a^{n-1}\right)}{\left(a^{n}-1\right) \ldots\left(a^{n}-a^{n-1}\right)} .
$$

(The terms in the series are the $q$-binomial polynomials, with $q=a$. The value of the $n$th term at $x=a^{m}$ with $a$ a prime number is the number of $n$-dimensional planes in an $m$-dimensional vector space over $\mathbb{Z} / a \mathbb{Z}$. If we had the regular binomial polynomials instead, the series would equal $2^{x}$ and in fact (over $\mathbb{C}$ ) $T\left(a^{z}\right)$ tends to $2^{z}$ as $a$ tends to 1 . It follows from Remark 2.2, and is not hard to see directly, that $T\left(a^{n}\right)$ is a polynomial in $a$ with integer coefficients for every $n \in \mathbb{N}$.)

We want to find an expression for the Taylor expansion of $T$ as a function of $x$. We start by proving the following.

LEMMa 6.1.

$$
T\left(a^{2} x\right)-2 T(a x)+(1-a x) T(x)=0 .
$$

Proof. First consider

$$
H(x)=T(a x)-T(x)=A+\sum_{n=2}^{\infty} \frac{(x-1)(x-a) \ldots\left(x-a^{n-2}\right)}{\left(a^{n}-1\right)\left(a^{n}-a\right) \ldots\left(a^{n}-a^{n-1}\right)} B,
$$

where

$$
\begin{aligned}
& A=\frac{a x-1}{a-1}-\frac{x-1}{a-1}=x, \\
& B=a^{n-1}(a x-1)-\left(x-a^{n-1}\right)=x\left(a^{n}-1\right) .
\end{aligned}
$$

Hence

$$
H(x)=x\left(1+\sum_{n=2}^{\infty} \frac{(x-1) \ldots\left(x-a^{n-1}\right)}{\left(a^{n}-1\right) \ldots\left(a^{n}-a^{n-1}\right)} \cdot \frac{1}{a^{n}}\right) .
$$

Now let $\Delta=T\left(a^{2} x\right)-2 T(a x)+(1-a x) T(x)$. Then

$$
\Delta=H(a x)-H(x)-a x T(x)=x\left(C+\sum_{n=2}^{\infty} \frac{(x-1) \ldots\left(x-a^{n-2}\right)}{\left(a^{n}-1\right) \ldots\left(a^{n}-a^{n-1}\right)} \cdot \frac{D}{a^{n}}\right),
$$


where

$$
\begin{aligned}
& C=-1+\frac{a x-1}{a-1}-\frac{x-1}{a(a-1)}-\frac{a(x-1)}{a-1}=\frac{-x+1}{a(a-1)}, \\
& D=a^{n}(a x-1)-\left(x-a^{n-1}\right)-\left(x-a^{n-1}\right)=-\left(x-a^{n-1}\right)+a^{n}\left(a^{n}-1\right) .
\end{aligned}
$$

Therefore,

$$
\begin{aligned}
\Delta= & x\left(-\frac{x-1}{a(a-1)}\right)-x \sum_{n=2}^{\infty} \frac{(x-1) \ldots\left(x-a^{n-1}\right)}{\left(a^{n}-1\right) \ldots\left(a^{n}-a^{n-1}\right) a^{n}} \\
& +x \sum_{n=2}^{\infty} \frac{(x-1) \ldots\left(x-a^{n-2}\right)}{\left(a^{n-1}-1\right) \ldots\left(a^{n-1}-a^{n-2}\right) a^{n-1}}=0
\end{aligned}
$$

and we are done.

Let

$$
T(x)=\sum_{n=0}^{\infty} t_{n}(a) x^{n}
$$

be the Taylor expansion of $T$ as a function of $x$. It is easy to see that our previous lemma is equivalent to the following recursion for the $t_{n}$ 's:

$$
t_{n}=\frac{a}{\left(a^{n}-1\right)^{2}} t_{n-1}
$$

from which it follows that

$$
T(x)=t_{0}\left(1+\sum_{n=1}^{\infty} \frac{a^{n}}{\left(a^{n}-1\right)^{2} \ldots(a-1)^{2}} x^{n}\right) .
$$

If we now evaluate this identity at $x=1$ we get

$$
t_{0}^{-1}=1+\sum_{n=1}^{\infty} \frac{a^{n}}{\left(a^{n}-1\right)^{2} \ldots(a-1)^{2}},
$$

and hence by an identity of Euler (see for example Hardy and Wright [12, Theorem 351])

$$
t_{0}=\prod_{n=1}^{\infty}\left(1-a^{-n}\right) .
$$

We have thus proved the following.

Proposition 6.2. The Taylor expansion of $T$ as a function of $x$ is given by

$$
T(x)=\prod_{n=1}^{\infty}\left(1-a^{-n}\right)\left(1+\sum_{n=1}^{\infty} \frac{a^{n}}{\left(a^{n}-1\right)^{2} \ldots(a-1)^{2}} x^{n}\right) .
$$


Notice that if we evaluate at $x=0$ we recover the classical identity

$$
\prod_{n=1}^{\infty}\left(1-a^{-n}\right)=1+\sum_{n=1}^{\infty} \frac{(-1)^{n}}{\left(a^{n}-1\right) \ldots(a-1)} .
$$

We next establish a property for all semidiffuse parades. To formulate our result we require some further notation. Suppose that $X=\left\{x_{0}, x_{1}, \ldots, x_{n}\right\}$ is an infinite proper sequence of real numbers. For each non-negative integer $n$ we set

$$
I_{n}=I_{n}(X)=\left[\min \left\{x_{0}, x_{1}, \ldots, x_{n}\right\}, \max \left\{x_{0}, x_{1}, \ldots, x_{n}\right\}\right]
$$

and

$$
I_{X}=\bigcup I_{n}(X)
$$

Thus $I_{n}$ is the convex closure of $\left\{x_{0}, \ldots, x_{n}\right\}$ in $\mathbb{R}$ and $I_{X}$ is the convex closure of $X$. In all the cases we consider we have $\left|x_{n}\right| \rightarrow \infty$ as $n \rightarrow \infty$ so that $I_{X}$ is either all of $\mathbb{R}$ or a halfline $(-\infty, c]$ or $[c, \infty)$ for some real $c$.

A real-valued function $g \in C^{\infty}\left(I_{X}\right)$ will be called univocal for $X$ at stage $n$ if $g^{(k)}$ is non-vanishing on $I_{k}(X)$ for all $k \geq n$. We will call $g$ univocal for $X$ if there is a non-negative integer $n$ such that $g$ is univocal for $X$ at stage $n$, and the least $n$ for which this holds will be called the stage of $g$ for $X$ and denoted $\operatorname{stag}(X, g)$.

A parade $X$ with $T_{X}$ entire will be called univocal if $T_{X}$ is univocal for $X$. We then define the stage of $X$ to be $\operatorname{stag}\left(X, T_{X}\right)$ and denote it $\operatorname{stag}(X)$.

Proposition 6.3. Let $X$ be a semidiffuse parade of integers. Then $X$ is univocal.

Proof. Choose positive constants $C$ and $\delta>1$ and a non-negative integer $M$ so large that, for $n \geq M$, the following inequalities hold:

$$
\begin{gathered}
\prod_{j=0}^{n-1}\left(1-\frac{\left|x_{j}\right|}{\left|x_{n}\right|}\right) \geq \frac{1}{C}, \quad \prod_{j=0}^{n-1}\left(1+\frac{\left|x_{j}\right|}{\left|x_{n}\right|}\right) \leq C, \\
\left|x_{n+1}\right| /\left|x_{n}\right| \geq \delta, \quad \delta>\exp (1 / n) .
\end{gathered}
$$

Let $S(i, k)$ denote the sum of all products of $i$ distinct terms from $\left\{x_{0}, x_{1}, \ldots, x_{k-1}\right\}$. The number of summands is $\left(\begin{array}{c}k \\ i\end{array}\right)$. Then for $k$ non-negative and $x \in \mathbb{R}$ we have

$$
\left(x_{k}-x_{0}\right)\left(x_{k}-x_{1}\right) \ldots\left(x_{k}-x_{k-1}\right) \phi_{X, k}(x)=\sum_{i=0}^{k}(-1)^{k-i} S(k-i, k) x^{i} .
$$

For $n \leq k$ we therefore have

$$
\left(x_{k}-x_{0}\right)\left(x_{k}-x_{1}\right) \ldots\left(x_{k}-x_{k-1}\right) \phi_{X, k}^{(n)}(x)=\sum_{i=n}^{k} \frac{(-1)^{k-i} S(k-i, k) i !}{(i-n) !} x^{i-n} \text {. }
$$


If $x \in I_{n}$ and $n \leq i \leq k$ then

$$
\left|S(k-i, k) x^{i-n}\right| \leq\left(\begin{array}{c}
k \\
k-1
\end{array}\right) r_{n} r_{n+1} \ldots r_{k-1}
$$

and hence, if $n \geq M$, we find that

$$
\begin{aligned}
\frac{r_{k}^{k}}{C}\left|\phi_{X, k}^{(n)}(x)\right| & \leq \frac{k !}{(k-n) !} \sum_{j=0}^{k-n}\left(\begin{array}{c}
k-n \\
j
\end{array}\right)\left|r_{n} r_{n+1} \ldots r_{k-1}\right| \\
& \leq \frac{k !}{(k-n) !} 2^{k-n}\left|r_{n} \ldots r_{k-1}\right| .
\end{aligned}
$$

Since $\left|\phi_{X, n}^{(n)}\right| \geq 1 /\left(C r_{n}^{n}\right)$ we have, for $x \in I_{n}$,

$$
\begin{aligned}
\frac{\left|T_{X}^{(n)}(x)-\phi_{X, n}^{(n)}\right|}{\left|\phi_{X, n}^{(n)}\right|} & \leq C^{2} \sum_{k=n+1}^{\infty} \frac{k ! 2^{k-n} r_{n}^{n+1} r_{n+1} \ldots r_{k-1}}{(k-n) ! r_{k}^{k}} \\
& \leq C^{2} \sum_{j=1}^{\infty} \frac{((n+j) !) 2^{j}}{j ! \delta^{j n+j(j+1) / 2}}
\end{aligned}
$$

We observe that

$$
\left(\frac{(n+j) !}{j !}\right) \leq \frac{n^{j}}{j !} \prod_{h=1}^{j}\left(1+\frac{h}{n}\right) \leq \frac{n^{j}}{j !} \exp \left(\sum_{h=1}^{j} \frac{h}{n}\right) \leq \frac{n^{j}}{j !} \exp \left(\frac{j(j+1)}{2 n}\right) .
$$

We have assumed $n$ so large that $\sqrt{\delta}>\exp (1 /(2 n))$. Hence

$$
\frac{\max \left\{\left|T_{X}^{(n)}(x)-\phi_{X, n}^{(n)}\right| \mid x \in I_{n}\right\}}{\left|\phi_{X, n}^{(n)}\right|} \leq C^{2} \sum_{j=1}^{\infty} \frac{(2 n)^{j}}{j ! \delta^{j n}}=C^{2}\left(\exp \left(\frac{2 n}{\delta^{n}}\right)-1\right) \rightarrow 0
$$

as $n \rightarrow \infty$. Hence $T^{(n)}$ is non-vanishing on $I_{n}$ for all sufficiently large $n$.

REMARKs. 1) The proof of Proposition 6.3 shows that the sign of $T^{(n)}$ on $I_{n}$ is the same as the sign of $\left(x_{n}-x_{0}\right)\left(x_{n}-x_{1}\right) \ldots\left(x_{n}-x_{n-1}\right)$. It follows that the envelope function $T_{X}$ of a semidiffuse procession $X$ shares with $2^{z}$ the property that all but at most finitely many Taylor coefficients are strictly positive.

2) Let $X$ be a univocal parade with envelope function $T=T_{X}$. If $f$ is a complex-valued function possessing $n$ derivatives on $I_{n}$ then the equality

$$
\frac{1}{V\left(x_{0}, \ldots, x_{n}\right)} \operatorname{det}\left(\begin{array}{ccccc}
1 & x_{0} & \ldots & x_{0}^{n-1} & f\left(x_{0}\right) \\
& \vdots & & \vdots & \vdots \\
1 & x_{n} & \ldots & x_{n}^{n-1} & f\left(x_{n}\right)
\end{array}\right)
$$




$$
\begin{aligned}
= & \int_{0}^{1} d t_{0} \int_{0}^{1} t_{0} d t_{1} \int_{0}^{1} t_{1} t_{0} d t_{2} \ldots \int_{0}^{1} t_{n-2} \ldots t_{0} d t_{n-1} \\
& \times f^{(n)}\left(x_{0}+t_{0}\left(x_{1}-x_{0}\right)+\ldots+t_{n-1} \ldots t_{0}\left(x_{n}-x_{n-1}\right)\right)
\end{aligned}
$$

is easily established by induction. This leads to the following alternative expression for the $c_{n}=c_{n}(X, f)$ of Proposition 4.5:

$$
\begin{aligned}
c_{n}=\frac{V_{n}(X)}{V_{n-1}(X)} \int_{0}^{1} d t_{0} \ldots \int_{0}^{1} t_{n-2} \ldots t_{0} d t_{n-1} & \\
& \times f^{(n)}\left(x_{0}+\ldots+t_{n-1} \ldots t_{0}\left(x_{n}-x_{n-1}\right)\right) .
\end{aligned}
$$

For each integer $n \geq \operatorname{stag}(X)$ set

$$
a_{n}(X, f)=\max \left\{\left|\frac{f^{(n)}(x)}{T_{X}^{(n)}(x)}\right|, x \in I_{n}(X)\right\} .
$$

For the envelope function $T$ one has $c_{n}(X, T)=1$ for all $n$. Hence by comparing integrands one sees that

$$
\left|c_{n}(X, f)\right| \leq a_{n}(X, f) .
$$

This estimate may be used to give a variant of Theorem 1.2 in which $f$ and $T$ are compared on the real line only: the growth hypothesis on $f$ in this variant is that $a_{n}(X, f)<1$ for all sufficiently large $n$. As the referee pointed out to us, this variant, in the case $X=\mathbb{N}$, may be deduced from the results of Pólya [16] or Selberg [18] mentioned in Section 1. Indeed, under the hypotheses, for any procession $X$, one finds that $M(f, r) / M(T, r)$ is bounded as $r \rightarrow \infty$. Results for other parades analogous to those of [16, $18]$ for $X=\mathbb{N}$ are not known. In the other direction, it is straightforward to deduce Pólya's original result (with the factor $\sqrt{r}$ ) from this variant. This may be accomplished using Cauchy's Theorem and Stirling's Formula.

\section{References}

[1] J.-P. Bézivin, Itération de polynômes et fonctions entières arithmétiques, Acta Arith. 68 (1994), 11-25. MR 95k:30057.

[2] —, Suites d'entiers et fonctions entières arithmétiques, Ann. Fac. Sci. Toulouse Math. 3 (1994), 313-334. MR 96a:11098.

[3] R. P. Boas, Comments on [15], in: George Pólya: Collected Papers, Vol. 1, R. P. Boas (ed.), M.I.T. Press, Cambridge, 1974, 771-773.

[4] N. Bourbaki, Commutative Algebra, Hermann, Paris, 1972.

[5] R. C. Buck, Integral valued entire functions, Duke Math. J. 15 (1948), 879-891.

[6] P. Bundschuh, A theorem of Gelfond via Schneider's method, in: New Trends in Probability and Statistics, F. Schweiger and E. Mantavicius (eds.), VSP, Utrecht, 1992, 9-15. 
[7] K. Ford, personal communication of 3 April 1998.

[8] A. O. Gel'fond, Sur les fonctions entières, qui prennent des valeurs entières dans les points $\beta^{n}, \beta$ est un nombre entier positif et $n=1,2,3, \ldots$, Mat. Sb. 40 (1933), 42-47 (in Russian; French summary).

[9] - Calculus of Finite Differences, authorised English translation of the third Russian edition, Hindustan Publishing Corporation, Delhi, 1971.

[10] R. R. Hall, On pseudopolynomials, Mathematika 8 (1971), 71-77.

[11] G. H. Hardy, On a theorem of Mr. G. Pólya, Proc. Cambridge Philos. Soc. 19 (1917), 60-63.

[12] G. H. Hardy and E. M. Wright, An Introduction to the Theory of Numbers, 5th ed., Clarendon Press, Oxford, 1979.

[13] A. Perelli and U. Zannier, On recurrent mod $p$ sequences, J. Reine Angew. Math. 348 (1984), 135-146. Math. Rev. 85g:11012.

[14] C. Pisot, Über ganzwertige ganze Funktionen, Jahresber. Deutsch. Math.-Verein. 52 (1942), 95-102. Math. Rev. 4, p. 270.

[15] G. Pólya, Ueber ganzwertige ganze Funktionen, Rend. Circ. Mat. Palermo 40 (1915), 1-16. Also in: Collected Papers, Vol. 1, R. P. Boas (ed.), M.I.T. Press, Cambridge, 1974, 1-16.

[16] - Über ganze ganzwertige Funktionen, Nachr. Ges. Wiss. Göttingen 1920, 1-10. Also in: Collected Papers, Vol. 1, 131-140.

[17] R. M. Robinson, Integer-valued entire functions, Trans. Amer. Math. Soc. 153 (1971), 451-468.

[18] A. Selberg, Über ganzwertige ganze transzendente Funktionen, Archiv for Math. og Naturvidenskab B. 44 (1941), 45-52. Also in: Collected Papers, Vol. 1, Springer, Berlin, 1989, 54-61.

[19] E. C. Titchmarsh, The Theory of Functions, 2nd ed., Oxford Univ. Press, 1939.

Department of Mathematics

University of Melbourne

Melbourne, Australia

E-mail: pila@ms.unimelb.edu.au
Department of Mathematics University of Texas at Austin

Austin, Texas 78712-1082

U.S.A.

E-mail: villegas@math.utexas.edu Web: http://www.ma.utexas.edu/users/villegas 